\title{
Bioanalysis
}

\section{Ensuring quality and compliance in outsourcing of bioanalysis of clinical biomarkers}

\begin{abstract}
"...the biomarker analysis field is still developing, and 'fit-for-purpose' approaches, as well as tiered 'quality' approaches are still evolving.'
\end{abstract}

First draft submitted: 14 January 2017; Accepted for publication: 2 February 2017;

Published online: 16 March 2017

Keywords: bioanalytical $\bullet$ clinical biomarkers $\bullet$ compliance $\bullet$ outsourcing

\section{Outsourcing \& clinical biomarkers}

Productivity and efficiency of pharmaceutical $\mathrm{R} \& \mathrm{D}$ has been a subject of multiple discussions and publications. Outsourcing is one of the common approaches pharmaceutical industry employs to address $R \& D$ challenges. Outsourcing in the pharma R\&D was initially started in 80 s as a cost-cutting measure. More recently, outsourcing is employed by large pharmaceutical companies for efficiency gains and allowing internal $\mathrm{R} \& \mathrm{D}$ functions to focus on innovation and core competency. In addition, outsourcing can facilitate the resource-capacity management (e.g., peak resource demands), project flexibility, access to specific instrumentation/technologies or regulatory compliance set up (e.g., CLIA or GLP). Small- to mid-size pharma companies are also following this outsourcing trend. Furthermore, some companies are putting outsourcing at the center of their business model. As a result, clinical outsourcing has significantly grown across late-stage clinical trials and into translational clinical studies.

Clinical biomarkers are another growing area of pharmaceutical $R \& D$. It is primarily driven by potential for personalized medicine, faster decision making and accelerating drug development. The aim of this editorial is to provide a holistic picture of clinical biomarker outsourcing, focusing on exploratory and molecular biomarker bioanalysis.

\section{Regulatory basis for clinical biomarker analysis}

Molecular biomarkers are a very broad class of biomolecules, which include metabolites, peptides, proteins, DNA and RNA, among others. Classification of clinical biomarkers can be quite complex and is a subject of debate [1]. However, from a regulatory bioanalysis standpoint, molecular biomarkers can be separated into biomarkers which contribute to the internal decision making and biomarkers supporting 'regulatory actions and label claims' $[2,3]$. It is also important to note that even biomarkers for internal decision making and exploratory biomarkers may be included in clinical study reports. As a result, exploratory biomarkers may have a certain health-authority exposure. This can also lead to a perception of 'high compliance risk' even for exploratory biomarkers.

From regulatory compliance perspectives, biomarker analysis has no specific healthauthority guideline to follow. The ambiguity of regulatory compliance of clinical biomarker bioanalysis can easily be illustrated by comparing molecular biomarker analysis versus regulated bioanalytical work (PK). For example, multiple guidance and White Papers exist that provide detailed guidance on the method validation and acceptance criteria for bioanalytical PK [3-5]. Furthermore, the industry has accumulated significant

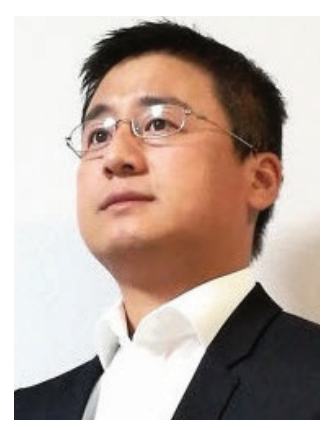

Tianke Wang

Biomarker Development, Translational Medicine, Novartis Institutes for BioMedical Research, Inc, Fabrikstrasse 10, Basel, Switzerland

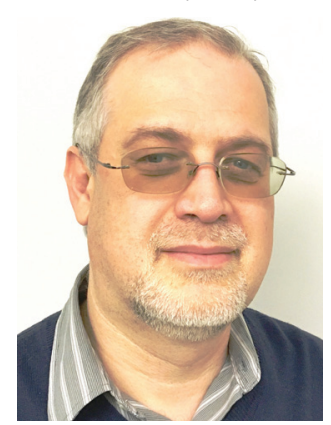

Arkady I Gusev

Biomarker Development, Translational Medicine, Novartis Institutes for BioMedical Research, Inc. 45 Sidney Street, Cambridge, MA 02139, USA Tel.: +1 6178713680 arkady.gusev@novartis.com 
experience in outsourcing bioanalytical and PK work as well as developing vendor qualification and vendor oversight processes.

In comparison, the actual practices of molecular biomarker bioanalysis vary and consist of a mixture of GLP, GCP, 'fit-for-purpose' and general quality considerations [6]. For example, GLP procedures primarily deal with laboratory quality and data integrity, and can be used for IT systems, experimental records, instrumentation, personal records (e.g., training, resume or job description) and for assay development and validation. The GCP ensures that patient safety and rights are not compromised and applicable to sponsor-laboratory communications, informed consent forms and data/patient privacy. The EMA paper [5] on laboratories that performed the analysis of clinical trial samples also brought the GCP consideration into the bioanalysis arena. However, this paper and its approaches are primarily applicable to $\mathrm{PK}$ bioanalysis rather than clinical biomarker analysis.

\section{"Strong partnership and communications can also help deal with the complexity of clinical operations and new regulations."}

\section{Fit-for-purpose biomarker assay}

A very common view on biomarkers is that clinical biomarker assays are not PK assays [7] and do not need to follow all requirements of regulated PK assays. The most common approach is to follow the 'fit-for-purpose' principles for the biomarker assay validation and characterization [8], a tiered approach to the assay validation and assay acceptance criteria $[6,9,10]$. The 'fit-forpurpose' assay refers to an assay that does not meet all current regulatory requirements for bioanalytical (PK) method validation and analysis but still demonstrates that the method is reliable for the intended application and data use. In other words, the method used for quantitative measurements in a given biological matrix is reliable and reproducible for the intended use [3]. The 'fit-for-purpose' principle does not preclude demonstrating that the assay is robust, repeatable, reliable and well characterized.

The 'fit-for-purpose' principle is directly related to clinical study questions, such as the purpose of the biomarker analysis. Therefore, the clinical study protocol and clinical questions define the intended use of biomarker data/assay requirements and analysis validation and acceptance criteria.

\section{Vendor qualification, partnership \& communication}

Outsourcing of clinical biomarkers analysis also has a number of unique challenges and opportunities. The first step is the qualification and selection of a vendor (i.e., specific CRO laboratory). The clinical requirements also add the necessity of meeting some regulatory compliance requirements, which can be a mixture of GLP and GCP considerations. Similar to the biomarker method validation and acceptance criteria, a tiered approach for the vendor qualification can be aligned with biomarkers for internal decision making (tier 1), and biomarkers supporting regulatory actions and label claims (tier 2). Tiered approaches to vendor qualification can also provide flexibility in the qualification process. For example, vendors supporting internal decision making may not require formal quality assurance (QA) audits and qualification may rely on a visit from the sponsor or even remote quality assessment.

Traditional selection of vendors based on costs and scientific expertise may not be optimal for outsourcing of clinical biomarker studies. The ambiguity and complexity of biomarker 'fit-for-purpose' considerations and the exploratory nature of some biomarkers require strong connections and communications between the sponsor's clinical team and CRO laboratory personnel. Communication is important for any regulatory and/ or clinical laboratory work, but it is one of the critical elements in outsourcing clinical biomarker studies. For example, the European Bioanalysis Forum [1] recommends active communication and involvement of laboratory bioanalysts to define the 'fit-for-purpose' assay requirements and acceptance criteria based on clinical study questions.

The necessity of a strong partnership and communication requires a different procurement approach for vendor selection (vs selecting new vendors based on the low-cost bids). Strategic and preferred vendor management approaches include: limiting a number of CROs, close oversight and monitoring of work and reusing the same $\mathrm{CRO}$ for new requests (i.e., new clinical biomarker analysis), re-enforcing the partnership, communication, and ensuring consistency and quality. It is important to note that strong partnership and close collaboration can lead to a significant streamlining of the assay validation and sample analysis and, in turn, lead to cost reduction [11]. It is also true that lack of partnership and communication can significantly increase the complexity and impact the quality of work and increase the final cost [12].

Strong partnership and communications can also help deal with the complexity of clinical operations (e.g., informed concept withdrawals) and new regulations (e.g., patience and data privacy).

\section{Oversight \& monitoring}

Sponsors of clinical studies have ultimate accountability for outsourced assays and analysis. Therefore, sci- 
entific monitoring and quality oversight of outsourced work are critical elements of the outsourcing model. Monitoring and oversight ensure scientific validly, data integrity, data quality, and allow to identify adverse trends, and take corrective and preventive actions for continuous improvement.

The US FDA 483 observations indicate that "method validation, acceptability of batch runs, events/deviations investigation/resolution, test specimen accountability and document change control" were the major categories of findings [13]. Therefore, the reliable and continuous monitoring and oversight of the integrity (i.e., chain of custody) of a laboratory's operation and process, the scientific validity of assay methodology and production data, constitute the foundation of quality for regulated bioanalysis [14]. For non-GxP research, effective vendor oversight is also a matter of some concern [15].

General vendor oversight can be satisfied by regular audits (or visits) supplemented by periodic (video or teleconference) reviews. QA audits can also be combined with scientific monitoring visits. At the clinical study and biomarker assay level, key oversight tasks include: review and approval of contracts, fit-for-purpose assay validation, informed consent form reports, sample reconciliation reports, data transfers, final data reports and so on. Close vendor oversight can also minimize the sponsor workload and resources. For example, regular data reconstruction, combined with the data security assessment of the vendor infrastructure allows the retention of raw data and relevant records at the vendor site, and avoid moving raw data and all documentation to the sponsor.

Scientific monitoring for biomarker analysis can also benefit from the risk-based tiered level of monitoring. A pragmatic approach to achieve consistency and efficiency is to categorize biomarker analyses into different risk levels (e.g., high, mid, low) and subsequently apply different levels of monitoring. This categorization can be based on several key criteria including the level of the assay validation, vendor experiences with the assay

\section{References}

1 Timmerman P, Herling C, Stoellner D et al. European Bioanalysis Forum recommendation on method establishment and bioanalysis of biomarker in support of drug development. Bioanalysis 4(15), 1883-1894 (2012).

2 Timmerman P. Biomarker assay validation: are we having the right discussion? Bioanalysis 8(22), 2299-2303 (2016).

3 FDA 2013 draft Guidance on Bioanalytical Method Validation.

www.fda.gov/downloads/drugs/guidancecompliance

4 U.S. Department of Health and Human Services Food and Drug Administration Center for Drug Evaluation and or biomarker clinical qualification. For example, a newly qualified vendor and new 'fit-for-purpose' assay would require in-depth scientific monitoring, whereas a preferred vendor and validated assay used in multiple clinical studies would need light monitoring.

Another effective approach in ensuring alignment between vendor and sponsor is to establish a common technical and planning document for analysis. A detailed bioanalytical plan can connect clinical study objectives and clinical questions with 'fit-for-purpose' assay performance and acceptance criteria. It can also include details for the oversight and scientific monitoring levels. A priori plan and acceptance criteria for biomarkers (except biomarker hypothesis-free screening) is also one of the most important regulatory compliance considerations [1].

\section{Conclusion}

The authors have tried to provide an overview and the key elements of outsourcing of clinical biomarker analysis. In general, clinical biomarkers supporting internal decision making or exploratory biomarkers may rely on sponsor-imposed quality standards and quality systems based on risk assessment and tiered processes. Biomarkers supporting registration end points and label claims are usually valid, qualified biomarkers, with analysis performed in regulated laboratories with robust and validated bioassays. However, the biomarker analysis field is still developing, and 'fit-for-purpose' approaches, as well as tiered 'quality' approaches are still evolving.

\section{Financial \& competing interests disclosure}

The authors have no relevant affiliations or financial involvement with any organization or entity with a financial interest in or financial conflict with the subject matter or materials discussed in the manuscript. This includes employment, consultancies, honoraria, stock ownership or options, expert testimony, grants or patents received or pending, or royalties.

No writing assistance was utilized in the production of this manuscript.

Research (CDER) Center for Veterinary Medicine (CVM). Guidance for Industry Bioanalytical Method Validation May 2001.

www.fda.gov/downloads/Drugs/Guidance/ucm070107.pdf

5 EMA GCP Inspectors Working Group Reflection paper for laboratories that perform the analysis or evaluation of clinical trial samples. EMA/INS/GCP/532137/2010 28 February 2012. www.ema.europa.eu/docs/en_GB/document_library

6 Hougton R, Gouty D, Allinson J et al. Recommendations on biomarker bioanalytical method validation by GCC. Bioanalysis 4(20), 2439-2446 (2012). 
7 Lowes S, Ackermann BL. AAPS and US FDA Crystal City VI workshop on bioanalytical method validation for biomarkers. Bioanalysis 8(3), 163-167 (2016)

8 Lee JW, Devanarayan V, Barrett YC et al. Fit-for-purpose method development and validation for successful biomarker measurement. Pharm. Res. 23(2), 312-28 (2006).

9 Timmerman P. Tiered approach revisited: introducing stage-appropriate or assay-appropriate scientific validation. Bioanalysis. 6(5), 599-604 (2014).

10 Hucker R, Watson C, James I, Vincent S, Muirhead D. Measuring soluble biomarkers in clinical trials: do tiered approaches to the analysis and validation of assays provide an answer to the fit-for-purpose challenge? Bioanalysis 6(5), 605-609 (2014).

11 Zimmer D. Bioanalytical outsourcing: the two sides of the medal. Bioanalysis 5(17), 2095-2099 (2013).
12 Clark GT. The importance of partnership when outsourcing exploratory bioanalysis. Bioanalysis 6(6), 733-736 (2014).

13 Chow F, Lum S, Ocampo A, Vogel P. Current challenges for FDA regulated bioanalytical laboratories for human (BA/BE) studies, part II: recent FDA inspection trends for bioanalytical laboratories using LC-MS/MS methods and FDA inspection readiness preparation. Qual. Assur. J. 11, 111-122 (2007).

14 He L. Conducting remote bioanalytical data monitoring and review based on scientific quality objectives. Bioanalysis 3(13), 1447-1456 (2011).

15 Volsen SG, Masson MM, Bongiovanni SA. Diligent process to help evaluate the quality of outsourced, non-GxP research projects. Quasar 128, 16-20 (2014). 